\title{
STUDY OF NONLINEAR WAVE PROCESSES IN PLASMAS USING THE FORMALISM OF A SPECIAL LORENTZ TRANSFORMATION FOR A SPACE-INDEPENDENT FRAME
}

\author{
S. N. PAUL" and B. CHAKRABORTY \\ Department of Mathematics \\ Jadavpur University \\ Calcutta - 700032, India \\ and

\section{DEBNATH} \\ Department of Mathematics \\ University of Central Florida \\ Orlando, Florida 32816
}

(Received Nay 11, 1984 and in revised form December 7, 1984)

ABSTRACT. A study is made of norilinear waves in plasmas using the formalism of a special Lorentz transformation for a space-independent frame, S'. This special transformation is used to transform the space-time dependent equations in a cold, relativistic, magnetized plasma to the $S^{\prime}$ frame. Then the transformed equations are employed to derive the expressions for the Lagrangian and the Hamiltonian in the $S^{\prime}$ frame. The Lagrangian and the Hamiltorian for a strong circularly polarized laser beam have also been obtained in the $S^{\prime}$ frame. The exact form of the nonlinear dispersion relation is derived for circularly polarized waves. Then the results for the frequency and the wave number shifts of these waves in a cold, magnetized relativistic plasma are obtained with some discussion on the nature of the frequency shifts. Finally, numerical results are presented for the radiation of Nd-glass laser in dense plasmas.

KEY WORDS AND PHRASES. Nonlinear waves in plasmas, relativistic plasma, Lorentz transformation

1980 MATHEMATICS SUBJECT CLASSIFICATION CODE. $76 \times 65$.

1. INTROLUCTION.

Using a special Lorentz transformation (SLT), the space-time dependent variables of ar electromagnetic wave in a plasma in the laboratory frame, $S$ become space iridependent in a moving frame, $S^{\prime}$ when the latter frame moves with a relative velocity $c^{2} / V, V$ is the group velocity of the wave. Winkles and Eldridge [1] first employed the SLT to obtain self-consistent solutions of the relativistic Vlasov- 
Näxwell equations, and have shown that a pure transverse wave cannot exist, but the coupled longitudinal field does necessarily appear. They also obtained a nonlinear dispersion relation correct up to the squares of the field amplitude. Thus the Lorentz transformation (LT) is found to be very useful because of the fact triat it can transform a system of nonlinear partial differential equations for a plasme into à set of ordinary differeritial equations.

Several áthors inciucing Clenımow [2-3], Chian and Clemmow [4], Kennàl and Pellat [5], Shih [6], Decoster [7], Lee and Lerche [8-11], Clemmow and Harding [12] have used the special Lorentz transformation to study nonlinear problems in plasma dynamics. On the cther hand, Akhiezer and Polovin [13], Wong [14], Wong and Lojko [15] have employed siriilar transformation relations for the investigation of nonliriear wave propagation in relativistic plasmas. Recently, Paul and Chakraborty [16-1]? developed the theory of transformations of nonlinear plasma equations, and extended it tc yield the nonlinear precessional rotation of an elliptically polarized, electromagnetic wave in an unmagnetized, cold, collisionless plasma in addition to the nonliriear shifts of wave parameters.

The main purpose of the present work is to develop the work of Winkles and Eldridge [1] for the transformation of field variables from a laboratory inertial frame, $S$ to the space independent frame, S'. Such a study is very useful for the investigation of several norilinear effects in plasmas. In particular, the study of self-action effects including self-focusing, self-steepening, self-phase modulation, self-precession is expected to be simpler by using the transformation relations for the S' frame for several reasons. First, some of the field variables become either constant or zero. For instance, the number density of electrons and the scalar potential become constant, and the oscillation of the magnetic field vanishes. Second, some nonlinear terms, which appear in the $S$ frame, vanish in the $S$ ' frame. Consequently, the rionlinear terms are fewer in number in the $S^{\prime}$ frame than in the $S$ frame.

Notivated by the above discussioris, we shall use the special Lorentz transformation to transform the space-time dependent equations in a cold, relativistic, magnetized plasma to the $S^{\prime}$ frame. These transformed equations are then employed to derive the expressions for the Lagrangian and the Hamiltonian for a strong circularly polarized laser beam in the $S^{\prime}$ frame. The exact expression for the nonlinear dispersion relation is derived for circularly polarized waves. Then the results for the frequency and the wave number shifts of the waves in a cold, magnetized relativistic plasma are obtained. Some attention is given to the nature of the frequency shift for different intensities of the wave and the static magnetic field. Finally, some numerical results for the radiation of $\mathrm{Nd}-\mathrm{glass}$ laser in dense plasmas are presented.

\section{BASIC EQUATIONS AND ASSUMPTIONS.}

We make the following assumptions:

(i) The plasma is cold, homogeneous and stationary, and is subject to a strong radiation with intensity less than $3 \times 10^{22}$ watts $/ \mathrm{cm}^{2}$ resulting in electron velocity 
becoming relativistic. The icn motion is negligibly small in comparison with the electron motion.

(ii) The forces due to other sources including gravitational and ponderomotive forces are also regligible.

With these assumptions, the basic equations of plasma in the S-frame are given by

$$
\begin{gathered}
{[D+(\underline{v} \cdot \underline{\nabla})] \mathrm{p}=-\mathrm{e} \underline{E}-\frac{\mathrm{e}}{\mathrm{c}}(\underline{\mathrm{v}} \times \underline{\mathrm{H}})} \\
\mathrm{DN}+\underline{\nabla} \cdot(\underline{N} \underline{\mathrm{v}})=0 \\
\underline{\nabla} \times \underline{E}=-\frac{1}{\mathrm{C}} D \underline{H} \\
\underline{\nabla} \times \underline{H}=-\frac{1}{\mathrm{C}} D \underline{E}-\frac{4 \pi \mathrm{e}}{\mathrm{C}}(\mathrm{Nv}) \\
\underline{\nabla} \cdot \underline{E}=-4 \pi e\left(N-N_{i}\right) \\
\underline{\nabla} \cdot \underline{H}=0
\end{gathered}
$$

where $\quad e=m_{c} r \underline{v}, \gamma=\left(1-\frac{v^{2}}{c^{2}}\right)^{-\frac{1}{2}}, D \equiv \frac{\partial}{\partial t}$;

and $N_{j}$ arid $N$ are the number densities of ions and electrons, $m_{0}$ and $-e$ are the rast miass aric charge of an electron, and other parameters have their usual meanings.

3. SPACE-INDEPENCENT FRAME AND TRANSFORMATION OF CUANTITIES $10 \mathrm{IT}$.

The Lorentz transformation from the $S$-frame to the $S^{\prime}-$ frame moving with a relative velocity $v_{0}$ parallel to the $z$-axis is given by

$$
t=\left(t^{\prime}+v_{0} z^{\prime} / c^{2}\right) \gamma_{0}, z=\left(z^{\prime}+v_{0} t^{\prime}\right) \gamma_{0}, x=x^{\prime}, y=y^{\prime},
$$

where $\gamma=\frac{1}{\left(1-\beta_{0}^{2}\right)^{\frac{1}{2}}}, \beta_{0}=\frac{V_{0}}{c}$

We follow Decoster [7] to obtain

and find from the relations (3.1) that

$$
\beta_{0}=\tanh \Psi_{0}
$$

$$
\left.\begin{array}{c}
\gamma_{0}=\cosh \psi_{0}, \beta_{0} \gamma_{0}=\sinh \psi_{0}, \\
t=t^{\prime} \cosh \psi_{0}+\frac{z^{\prime}}{c} \sinh \psi_{0}, \\
z=z^{\prime} \cosh \psi_{0}+c t^{\prime} \sinh \psi_{0}, \\
x=x^{\prime}, y=y^{\prime} .
\end{array}\right\}
$$

It is obvious that $\psi_{0}$ is the hyperbolic angle for the $S^{\prime}$-systeni relative to the $S$-system. The reverse transformation from $S^{\prime}$ to $S$ is obtained by changing the sign of $V_{0}$ or $\psi_{0}$, that is,

$$
\left.\begin{array}{l}
t^{\prime}=t \cosh \psi_{0}-\frac{z}{c} \sinh \psi_{0}, \\
z^{\prime}=z \cosh \psi_{0}-c t \sinh \psi_{0} \\
x^{\prime}=x, y^{\prime}=y
\end{array}\right\}
$$


These relations show that the wave phase

$$
\omega t-k z=\left(\omega \cosh \psi_{0}-k c \sinh \psi_{0}\right) t^{\prime}-\left(k \cosh \psi_{0}-\frac{\omega}{c} \sinh \psi_{0}\right) z^{\prime},
$$

where $k$ and $\omega$ are the constant wave number ard wave frequency respectively of an electromagnetic wave.

Followirg Winkles and Eldridge [1] we consider the transformation from $S$ to $S^{\prime}$ at the velocity $V_{0}=k c^{2} / \omega$ to obtain the phase velocity

$$
v=\omega / k=c^{2} / v_{0}
$$

Then (3.6) reduces to

$$
\omega t-k z=\omega\left(1-\beta_{0}\right)^{\frac{1}{2}} \cdot t^{\prime}
$$

This transformation enables us to change the field variables from the space-time dependent $S$ frame to the space-independent $S^{\prime}$-frame of primed variables. It is to be noted here that for transverse waves, the phase velocity $V(=\omega / k ;>c$ and so $V_{c}<c$. Therefore the velocity of $S^{\prime}$ relative to $S$ is not unphysical.

Feplacing $t^{\prime}$ by $T$ we can write

$$
\begin{aligned}
& \left.\begin{array}{l}
\omega^{\prime} T=\omega t-k z \\
v_{z}=\dot{z}=v-\frac{V}{\gamma_{0}} \frac{\partial T}{\partial t}
\end{array}\right\} \\
& \left.\begin{array}{l}
\omega^{\prime}=\omega\left(1-\beta_{0}\right)^{\frac{1}{2}}=\omega\left(1-\frac{c^{2}}{v^{2}}\right)^{\frac{1}{2}}=\omega / \gamma_{0} \\
\gamma_{0}^{2}=1 /\left(1-\frac{c^{2}}{v^{2}}\right)=1 /\left(1-\frac{v_{0}^{2}}{c^{2}}\right)
\end{array}\right\} \\
& \omega t-k z=\omega\left(1-\frac{c^{2}}{v^{2}}\right)^{\frac{1}{2}} \cdot T \\
& \frac{\partial}{\partial t}=\gamma_{0} \frac{\partial}{\partial T}, \frac{\partial}{\partial z}=-\frac{\gamma_{0}}{V} \frac{\partial}{\partial T}
\end{aligned}
$$

Akhiezer et àl. [18] considered a linear transformation rule

$$
\omega T=\omega t-k z, V=\omega / k \text {, }
$$

to solve the plasma equations (2.1) to (2.6) neglecting collision effect for some ronlinear problems. Boyd and Sanderson [19] considered the special value, $v=c$ for some irivestigations.

The transformation of some field variables from the $S$-frame to the $S^{\prime}$-frame is given by

$$
\begin{aligned}
& v_{x}=v_{x}^{\prime} / \gamma_{0}\left(1+\beta_{0} v_{z}^{\prime} / c\right)=\left(c v_{x}^{\prime}\right) /\left(c \cosh \psi_{0}+v_{z}^{\prime} \sinh \psi_{0}\right), \\
& v_{y}=v_{y}^{\prime} / \gamma_{0}\left(1+\beta_{0} v_{z}^{\prime} / c\right)=\left(c v_{y}^{\prime}\right) /\left(c \cosh \psi_{0}+v_{z}^{\prime} \sinh \psi_{0}\right), \\
& v_{z}=\left(v_{z}^{\prime}+\beta_{0} c\right) /\left(1+\beta_{0} v_{z}^{\prime} / c\right)=c\left(v_{z}^{\prime} \cosh \psi_{0}+c \sinh \psi_{0}\right) /\left(c \cosh \psi_{0}+v_{z}^{\prime} \sinh \psi_{0}\right) \\
& E_{x}=\gamma_{0}\left(E_{x}^{\prime}+\beta_{0} H_{y}^{\prime}\right)=E_{x}^{\prime} \cosh \psi_{0}+H_{y}^{\prime} \sinh \psi_{0}^{\prime}, \\
& \left.E_{y}=\gamma_{0}\left(E_{y}^{\prime}-\beta_{0} H_{x}^{\prime}\right)=E_{y}^{\prime} \cosh \psi_{0}-H_{x}^{\prime} \sinh \psi_{0}^{\prime}\right\} \\
& E_{z}=E_{z}^{\prime} \\
& H_{x}=\gamma_{0}\left(H_{x}^{\prime}-\beta_{0} E_{y}^{\prime}\right)=\left(H_{x}^{\prime} \cosh \psi_{0}-E_{y}^{\prime} \sinh \psi_{0}^{\prime}\right) \\
& \left.H_{y}=\gamma_{0}\left(H_{y}^{\prime}+\beta_{0} E_{x}^{\prime}\right)=H_{y}^{\prime} \cosh \psi_{0}+E_{x}^{\prime} \sinh \psi_{0}^{\prime}\right\} \\
& H_{z}=H_{z}^{\prime}
\end{aligned}
$$


The transformation of mass is

$$
m=m^{\prime} r_{0}\left(1+\beta_{0} v_{z}^{\prime} / c\right)
$$

as niay be seen frum Hughes arid Young [20, pi8-19] where $m_{0}$ (but not $m$ ) is the rest mass. The momentum components are transformed into the form

$$
\left.\begin{array}{c}
P_{x}=m v_{x}=m^{\prime} \gamma_{0}\left(1+\beta_{0} v_{z}^{\prime} / c\right) \frac{c}{\left(c \cosh \psi_{0}+v_{z}^{\prime} \sinh \psi_{0}^{\prime}\right.}=m^{\prime} v_{x}^{\prime}=P_{x}^{\prime}, \\
P_{y}=m v_{y}=m^{\prime} v_{y}^{\prime}=P_{y}^{\prime}, \\
P_{z}=m v_{z}=m^{\prime} \gamma_{0}\left(1+\beta_{0} v_{z}^{\prime} / c\right) \frac{c\left(v_{z}^{\prime} \cosh \psi_{0}+c \sinh \psi_{0}\right)}{\left(c \cosh \psi_{0}+v_{z}^{\prime} \sinh \psi_{0}\right)} \\
=P_{z}^{\prime} \gamma_{0}+m^{\prime} c \beta_{0} \gamma_{0}=\gamma_{0}\left(P_{z}^{\prime}+m_{0} v_{0} \gamma^{\prime}\right)
\end{array}\right\}
$$

Lefining now the momentum like quantities $q$ and $q^{\prime}$ as

$$
\begin{aligned}
& q=\left(m_{0}^{2} c^{2}+p^{2}\right)^{\frac{1}{2}}, q^{\prime}=\left(m_{0}^{2} c^{2}+p^{\prime}\right)^{\frac{1}{2}}, \\
& q^{\prime 2}=m_{0}^{2} c^{2}+\frac{m_{0}^{2} v^{\prime 2}}{\left(1-v^{2} / c^{2}\right)}=\frac{m_{0}^{2} c^{2}}{\left(1-v^{\prime}{ }^{2} / c^{2}\right)}=m^{\prime} c^{2}
\end{aligned}
$$

where $V^{\prime}$ is the velocity of the $S^{\prime}$-frame relative to the rest system $S_{0}$. So we have

$$
\begin{aligned}
P_{z} & =P_{z}^{\prime} \cosh \psi_{0}+q^{\prime} \sinh \psi_{0}, \\
q & =m c=m^{\prime} \gamma_{0} c\left(1+\beta_{0} v_{z}^{\prime} / c\right)=m^{\prime} \gamma_{0}\left(c+\beta_{0} v_{z}^{\prime}\right) \\
& =\gamma_{0}\left(q^{\prime}+P_{z}^{\prime} B_{0}\right)=q^{\prime} \cosh \psi_{0}+P_{z}^{\prime} \sinh \psi_{0}
\end{aligned}
$$

If $N^{\prime}, N^{\prime}, N_{0}$ are the symbols for the number density in the three systems $S, S^{\prime}, S_{0}$ respectively, then again following Hughes and Young [20] we get

$$
N^{\prime}=N_{0} /\left(1-V \cdot 2 / c^{2}\right)^{\frac{1}{2}}
$$

so we can write

$$
m_{0} N^{\prime}=m_{0} N_{0} /\left(1-V^{2} / c^{2}\right)^{\frac{1}{2}}=m 1^{\prime} N_{0}
$$

Similarly, we can also write

$$
\begin{gathered}
m_{0} N=m N_{0} \\
\frac{N^{\prime}}{N}=\frac{m^{\prime}}{m}=\frac{m^{\prime} c}{m c}=\frac{q^{\prime}}{q}
\end{gathered}
$$

Since $\underline{A}$ and $\phi$ form a four vector (A is vector potential and $\phi$ is the scalar potential!, and we can write

$$
\left.\begin{array}{l}
A_{z}=\gamma_{0}\left(A_{z}^{\prime}+B_{0} \phi^{\prime}\right)=A_{z}^{\prime} \cosh \psi_{0}+\phi^{\prime} \sinh \psi_{0}, \underline{A}_{\perp}=A_{\perp} \\
\phi=\gamma_{0}\left(B_{0} A_{z}^{\prime}+\phi^{\prime}\right)=\phi^{\prime} \cosh \psi_{0}+A_{z}^{\prime} \sinh \psi_{0}
\end{array}\right\}
$$

The reverse transformation is obtained by replacing $\psi_{0}$ by $-Y_{0}$.

Equation (3.21) gives

$$
N_{0}=N^{\prime} \operatorname{sech} \psi^{\prime}=\text { constant }
$$

and (3.25) can be written as

$$
N=N^{\prime} q / q^{\prime}=N_{0} q \cosh \psi^{\prime} /\left\{q \cosh \left(\psi^{\prime}-\psi\right)-P_{2} \sinh \left(\psi^{\prime}-\Psi\right)\right\}
$$

Here $\psi$ is the hyperbolic angle for the S-system relative to the $S_{0}$ frame and $\psi^{\prime}$ is the same for the $S^{\prime}$ system. Therefore, in the S-system, the number density is not coristant, and the electron and ion densities are not necessarily equal. 
4. TRANSFORMATION CF FIELD EQUATIONS TO THE SPACE INDEPENDENT FRAME

Using the transformation relations of section 3, we obtain from (2.1) for the $x$-component

$$
\begin{aligned}
& r_{0}\left(1-\frac{v_{z}^{\prime}}{V}\right) \frac{\partial P_{x}^{\prime}}{\partial T}=\left\{\gamma_{0} V-v_{0}+\left(\gamma_{0}-1\right) v_{z}^{\prime}\right\}\left(\frac{\partial P_{x}^{\prime}}{\partial T}\right) / V\left(1+\beta_{0} v_{z}^{\prime} / c\right) \\
& =-e \gamma_{0}\left(E_{x}^{\prime}+\beta_{0} H_{y}^{\prime}\right)+(e / c)\left(v_{z}^{\prime}+v_{0}\right) \gamma_{0}\left(H_{y}^{\prime}+\beta_{0} E_{x}^{\prime}\right) /\left(1+\beta_{0} v_{z}^{\prime} / c\right)-e v_{y}^{\prime} H_{z}^{\prime} / \gamma_{0} c\left(1+\beta_{0} v_{z}^{\prime} / c\right) \\
& =-\frac{\beta_{0} c E_{x}^{\prime}\left\{\gamma_{0} V-v_{0}+\left(\gamma_{0}-1\right) v_{z}^{\prime}\right\}}{c\left(1+\beta_{0} v_{z}^{\prime} / c\right)}-\frac{e H_{y}^{\prime}\left\{v_{0}\left(\gamma_{0}-1\right)+\left(\beta_{0}^{\prime} \gamma_{0}-1\right) v_{z}^{\prime}\right\}}{c\left(1+\beta_{0} v_{z}^{\prime} / c\right)}-\frac{E v_{y}^{\prime} H_{z}^{\prime}}{\gamma_{0} c\left(1+\beta_{0} v_{z}^{\prime} / c\right)}
\end{aligned}
$$

ihis equation ciar, be simplified to the form

$$
\begin{aligned}
\frac{\partial P_{x}^{\prime}}{\partial T}=-e E_{x}^{\prime}-\frac{e H_{y}^{\prime} V}{c\left(\gamma_{0} V-V_{0}\right)}\left[V_{0}\left(\gamma_{0}-1\right)\right. & \left.-\frac{V_{z}^{\prime}}{\gamma_{0}^{3}\left\{\gamma_{0} V-V_{0}+\left(\gamma_{0}-1\right) v_{z}^{\prime}\right\}}\right] \\
& -\frac{e V V_{y}^{\prime} H_{z}^{\prime}}{\left.\gamma_{0} c \gamma_{0} V-V_{0}+\left(\gamma_{0}-1\right) v_{z}^{\prime}\right\}}
\end{aligned}
$$

Similarly from (2.1) the equation for $P_{y}^{\prime}$ becomes

$$
\begin{aligned}
\frac{\partial P_{y}^{\prime}}{\partial T}=-e E_{y}^{\prime}+\frac{e H_{x}^{\prime} V}{c\left(\gamma_{0} V-V_{0}\right)}\left[V_{0}\left(\gamma_{0}-1\right)\right. & \left.-\frac{V v_{z}^{\prime}}{\gamma_{0}^{3}\left\{\gamma_{0} V-V_{0}+\left(\gamma_{0}-1\right) v_{z}^{\prime}\right\}}\right] \\
& +\frac{e V v_{x}^{\prime} H_{z}^{\prime}}{\gamma_{0} c\left\{\gamma_{0} V-V_{0}+\left(\gamma_{0}-1\right) v_{z}^{\prime}\right\}}
\end{aligned}
$$

Now, the equation for $P_{Z}^{\prime}$ obtained from (2.1) is

$$
\frac{\partial P_{z}^{\prime}}{\partial T}+m_{0} V_{0} \frac{\partial \gamma^{\prime}}{\partial T}=-\frac{e V i E_{z}^{\prime}+B_{0}\left(\underline{B}^{\prime} \cdot \underline{E}^{\prime}\right)+\left(\underline{\beta}^{\prime} X \underline{H}^{\prime}\right) z^{j}}{\gamma_{0}\left[\gamma_{0} V-V_{0}+\left(\gamma_{0}-1\right) V_{z}^{\prime}\right.},
$$

where

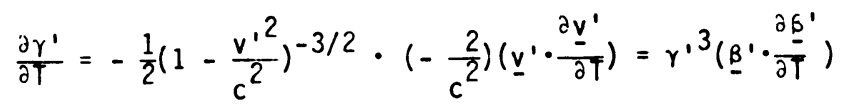

Since $B=\underline{p} /\left(p^{2}+m_{0}^{2} c^{2}\right)^{\frac{1}{2}}$, arid $p^{2}+m_{0}^{2} c^{2}=m_{0}^{2} c^{2} \gamma^{2}$, we obtain

$$
\frac{\partial \underline{B}}{\partial T}=\frac{1}{m_{0} c r} \frac{\partial \underline{P}}{\partial T}-\frac{\underline{P}(\underline{P} \cdot \partial \underline{P} / \partial T)}{m_{0}^{3} c^{2} \gamma^{3}} \text {. }
$$

Therefore,

$$
\left(\underline{\beta^{\prime}} \cdot \frac{\partial \underline{\beta}^{\prime}}{\partial T}\right)=\frac{\left(\underline{p}^{\prime} \cdot \partial \underline{p}^{\prime} / \partial T\right)}{\left(m_{0} c \gamma^{\prime}\right)^{2}}-\frac{\underline{P}^{\prime}{ }^{2}\left(\underline{p}^{\prime} \cdot \partial \underline{p}^{\prime} / \partial T\right)}{\left(m_{0} c \gamma^{\prime}\right)^{4}}=\frac{1}{m_{0}^{2} c^{2} \gamma^{\prime}} \quad\left(\underline{p}^{\prime} \cdot \frac{\partial \underline{p}^{\prime}}{\partial T}\right)
$$

Hence

$$
\frac{\partial P_{Z}^{\prime}}{\partial T}+\frac{V_{0}}{m_{0} c^{2} \gamma^{\prime}}\left(\underline{P}^{\prime} \cdot \frac{\partial \underline{P}^{\prime}}{\partial T}\right)=\frac{\operatorname{eV}\left\{E_{Z}^{\prime}+\beta_{0}\left(\underline{B}^{\prime} \cdot \underline{E}^{\prime}\right)+\left(\underline{B}^{\prime} x \underline{H}^{\prime}\right) z^{\}}\right.}{\gamma_{0}\left(\gamma_{0} V-V_{0}+\left(\gamma_{0}-1\right) v_{z}^{\prime}\right\}}
$$

Multiplying (4.2) by $P_{x}^{\prime},(4.3)$ by $P_{y}^{\prime}$ and $(4.8)$ by $P_{z}^{\prime}$ and then adding, we get after simplification 


$$
\begin{aligned}
& \left(\underline{P}^{\prime} \cdot \frac{\partial \underline{P}^{\prime}}{\partial T}\right)=-\frac{\operatorname{eV}\left(P_{x}^{\prime} E_{x}^{\prime}+P_{y}^{\prime} E_{y}^{\prime}\right)\left(\gamma_{0}\left(\gamma_{0} V-v_{0}\right)+v_{z}^{\prime}\left(\gamma_{0}^{2}-\gamma_{0}+1\right)\right\}}{\gamma_{0}\left(V+v_{z}^{\prime}\right)\left(\gamma_{0} V-v_{0}+\left(\gamma_{0}-1\right) v_{z}^{\prime}\right\}} \\
& -\frac{e V P_{z}^{\prime} E_{z}^{\prime}}{\gamma_{0}\left\{\gamma_{0} V-V_{0}+\left(\gamma_{c}-1\right) v_{z}^{\prime}\right\}} \\
& -\frac{\left.e v^{2}\left(\underline{p}^{\prime} x \underline{H}^{\prime}\right)_{z} v_{z}^{\prime}\left(v-\gamma_{0}^{2} v_{0}+\gamma_{0}^{4} v\right)+v_{\gamma_{0}}^{3}\left(\gamma_{c} v-v_{0}\right)\right\}}{\gamma_{0}^{3} c\left(v+v_{z}^{\prime}\right)\left(\gamma_{0} v-v_{0}\right)\left(\gamma_{0} v-v_{0}+\left(\gamma_{0}-1\right) v_{z}^{\prime}\right\}} \\
& -\frac{\mathrm{eV}\left(\underline{P}^{\prime} x \underline{v}^{\prime}\right){ }_{z} H_{z}^{\prime}}{C \gamma_{0}\left\{\gamma_{0} V-V_{0}+\left(\gamma_{0}-1\right) v_{z}^{\prime}\right\}}
\end{aligned}
$$

Therefore, the equation for $P_{z}^{\prime}$ becomes

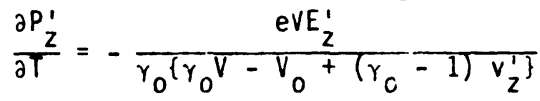

$$
\begin{aligned}
& +\frac{e\left(P_{x}^{\prime} E_{x}^{\prime}+F_{y}^{\prime} E_{y}^{\prime}\right)\left\{V\left(\gamma_{0}^{2} V-\gamma_{0} V_{0}-V_{0}\right)+v_{z}\left(V\left(\gamma_{0}^{2}-\gamma_{0}+1\right)-V_{0}\right)\right\}}{m_{0} \gamma_{0} \gamma^{\prime} V_{0}\left(V-v_{z}^{\prime}\right)\left\{\gamma_{0} V-V_{0}+\left(\gamma_{0}-1\right) v_{z}^{\prime}\right\}} \\
& +\frac{e V P_{z}^{\prime} E_{z}^{\prime}}{m_{0} \gamma_{0}^{3} \gamma^{\prime} v_{0}\left\{\gamma_{0} V-v_{0}+\left(\gamma_{0}-1\right) v_{z}^{\prime}\right\}} \\
& +\frac{\left.e\left(\underline{p}^{\prime} x \underline{H}^{\prime}\right) v_{2} v^{2} v_{z}^{\prime}\left(v+v_{\gamma_{0}}^{6}-v_{0} \gamma_{0}^{5}\right)+\gamma_{0}^{4}\left(\gamma_{0} v_{0}-v_{0}\right)^{2}\right\}}{c m_{0} \gamma_{0}^{5} \gamma^{\prime} v_{0}\left(v+v_{z}^{\prime}\right)\left(\gamma_{0} v-v_{0}\right)\left\{\gamma_{0} v-v_{0}+\left(\gamma_{0}-1\right) v_{z}^{1}\right\}} \\
& +\frac{\epsilon V V_{0}\left(\underline{P}^{\prime} x \underline{v}^{\prime}\right)_{z} H_{z}^{\prime}}{m_{0} c^{3} \gamma_{0} \gamma^{\prime}\left\{\gamma_{0} V-V_{0}+\left(\gamma_{0}-1\right) v_{z}^{\prime}\right\}}
\end{aligned}
$$

The equation of continuity (2.2) assumes the form

$$
\gamma_{0} \frac{\partial}{\partial T}\left(\frac{N^{\prime} \gamma}{\gamma}\right)-\frac{\gamma_{0}}{V} \frac{\partial}{\partial T^{\prime}}\left\{\frac{N^{\prime} \gamma}{\gamma^{\prime}} \cdot \frac{C\left(\beta_{z}^{\prime}+\beta_{0}\right)}{\left(1+\beta_{0} \beta_{z}^{\prime}\right)}\right\}=0
$$

It can be written as

$$
\gamma_{0} \frac{\partial}{\partial T}\left[\frac{N^{\prime}\left(1-\beta_{0}^{2}\right)^{\prime}}{\beta_{0}}\right]=0 .
$$

And so, $N^{\prime}=N_{0}$, where $N_{0}$ is a constant.

Equation for $H_{x}^{\prime}$, obtained from (2.3), is given by

$$
-\frac{1}{V} \frac{\partial}{\partial T}\left(E_{y}^{\prime}-B_{0} H_{x}^{\prime}\right)=\frac{1}{c} \frac{\partial}{\partial T}\left(H_{x}-B_{0} E_{y}^{\prime}\right)
$$

It can be written as

$$
\left(\frac{1}{\beta_{0}}-\beta_{0}\right) \frac{\partial H_{x}^{\prime}}{\partial T}=0
$$

So,

$$
H_{x}^{\prime}=\text { constant }
$$

And the equation for $H_{y}^{\prime}$, obtained from equation (2.3), is given by

Hence,

$$
\frac{\partial}{\partial T}\left[E_{x}^{\prime}\left(1-\frac{E_{0} V}{c}\right)+H_{y}^{\prime}\left(B_{0}-\frac{V}{c}\right)\right]=0
$$

The $x$-component of equation (2.4) is

$$
H_{y}^{\prime}=\text { constant. }
$$


Or

$$
\begin{aligned}
-\frac{\gamma_{0}^{2}}{V} \frac{\partial}{\partial T}\left(H_{y}^{\prime}+\beta_{0} E_{x}^{\prime}\right) & =-\frac{\gamma_{0}^{2}}{C} \frac{\partial}{\partial T}\left(E_{x}^{\prime}+\beta_{0} H_{y}^{\prime}\right)+\frac{4 \pi e N_{0} V_{x}^{\prime}}{C \gamma_{0}\left(1+\beta_{0} B_{z}^{\prime}\right)} . \\
\frac{\partial E_{x}^{\prime}}{\partial T} & =\frac{4 \pi e N_{0} V_{x}^{\prime}}{\gamma_{0}\left(1+\beta_{0} \beta_{z}^{\prime}\right)} .
\end{aligned}
$$

Similarly, the $y$ - and z-components of equation (2.4) become

$$
\frac{\partial E_{y}^{\prime}}{\partial T}=\frac{4 \pi e N_{0} v_{y}^{\prime}}{\gamma_{0}\left(1+\beta_{0} \beta_{z}^{\prime}\right)} \text {. }
$$

and

$$
\frac{\partial E_{z}^{\prime}}{\partial T}=\frac{4 \pi e N_{0}\left(v_{z}^{1}+v_{0}\right)}{r_{0}\left(1+E_{0} B_{z}^{\prime}\right)^{-}}
$$

We shall now expand $1 /\left(1+\beta_{0} B_{z}^{\prime}\right)$ in powers of $\beta_{z}^{\prime}$. Since $v_{z}=0$ when $v_{z}^{\prime}=-v_{0}$, we put $v_{z}=-v_{0}+\delta v_{z}^{\prime}$ to avoid a physical impossibility. Therefore,

$$
\frac{1}{\gamma_{0}\left(1+\beta_{0} \beta_{z}^{\prime}\right)}=\gamma_{0}^{2}\left[1-\gamma_{0}^{2}\left(\frac{\delta v_{z}}{V}\right)+\gamma_{0}^{4}\left(\frac{\delta v_{z}^{\prime}}{V}\right)^{2}-\ldots\right]
$$

So, the veiocity components becone

$$
\begin{aligned}
& v_{x}=v_{x}^{\prime} \gamma_{0}\left[1-\gamma_{0}^{2}\left(\frac{\delta v_{z}^{\prime}}{v}\right)+\gamma_{0}^{4}\left(\frac{\delta v_{z}^{\prime}}{v}\right)^{2}-\ldots\right] \\
& v_{y}=v_{y}^{\prime} \gamma_{0}\left[1-\gamma_{0}^{2}\left(\frac{\delta v_{z}^{\prime}}{v}\right)+\gamma_{0}^{4}\left(\frac{\delta v_{z}^{\prime}}{v}\right)^{2}-\ldots\right] \\
& v_{z}=\gamma_{0}^{2} \delta v_{z}^{\prime}\left[1-\gamma_{0}^{2}\left(\frac{\delta v_{z}^{\prime}}{v}\right)+\gamma_{0}^{4}\left(\frac{\delta v_{z}^{\prime}}{v}\right)^{2}-\ldots\right]
\end{aligned}
$$

where $v_{x}^{\prime}$ and $v_{y}^{\prime}$ are the first order velocity components in the directions parallel to $O X$ and $O Y$ respectively, $\delta v_{z}^{\prime}$ is the second order velocity component along $-0 z$ in the $S^{\prime}$-frame.

The continuity equation (2.2) gives

$$
\begin{aligned}
N=N_{0}+\delta N^{\prime} & =N_{0} /\left(1-\gamma_{0}^{2} \delta V_{z}^{\prime} / V\right) \\
\delta N^{\prime} & =N_{0} \gamma_{0}^{2} \delta v_{z}^{\prime} / V,
\end{aligned}
$$

and so

where $N_{0}$ is a constant, and higher powers of $\delta v_{z}^{\prime}$ are neglected.

Therefore, equations (4.20)-(4.22) for the electric field components become

$$
\begin{aligned}
\frac{\partial E_{x}^{\prime}}{\partial T}=\frac{4 \pi e}{r_{0}} \cdot \frac{\left(N_{0}+\delta N^{\prime}\right) v_{x}^{\prime}}{\left(1+\beta_{0} B_{z}^{\prime}\right)^{2}}=\frac{r_{0} m_{0} \omega_{e}^{2}}{e} v_{x}^{\prime}, \\
\frac{\partial E_{y}^{\prime}}{\partial T}=\frac{r_{0}^{m_{0} \omega_{p}^{2} p}}{e} v_{y}^{\prime}, \\
\frac{\partial E_{z}^{\prime}}{\partial T}=\frac{r_{0}^{m_{0} \omega_{p}^{2}}}{e} \delta v_{z}^{\prime},
\end{aligned}
$$

where $\omega_{p}=\left(4 \pi N_{0} e^{2} / m_{0}\right)^{\frac{1}{2}}, \omega_{p}$ being the electron plasma frequency. Again from the transformation relations $(3.14 \mathrm{abc})$ and $(3.15 \mathrm{abc})$, neglecting the transverse magnetic fitld components in the $S^{\prime}$-frame, we obtain from the transformation relations

$$
\begin{aligned}
& E_{x}=\gamma_{0} E_{x}^{\prime}, E_{y}=\gamma_{0} E_{y}^{\prime}, E_{z}=E_{z}^{\prime} \\
& H_{x}=-\gamma_{0} B_{0} E_{y}^{\prime}, H_{y}=-\gamma_{0} B_{0} E_{x}^{\prime}, H_{z}=H_{z}^{\prime}=H_{0}
\end{aligned}
$$




\section{THE LAGRANGIAN AND HAMILTONIAN IN THE SPACE-INDEPENDENT FRAME}

Following Lindau and Lifshitz [21], the expressions for the Lagrangian and Hamiltoniar in plasmas in the labcratory frame are given by

$$
\begin{gathered}
\varepsilon=-N_{0}\left[m_{0} c^{2}\left(i-\frac{v^{2}}{c^{2}} i^{\frac{1}{2}}+\frac{e}{c}(A-\underline{v})+e \phi\right]+\frac{E^{2}-H^{2}}{8 \pi}\right. \\
\left.H^{\prime}=N_{0} \frac{v}{\left(1-\frac{v^{2}}{c^{2}}\right)^{\frac{1}{2}}}-\frac{e}{c} \underline{A}\right]-\varepsilon
\end{gathered}
$$

where the lector ard scalar potentials $\underline{A}$ anc $\phi$ respectively are defined by

$$
\begin{gathered}
\underline{H}=\operatorname{curl} \underline{A} \\
\underline{E}=-\frac{1}{c} \frac{\partial \underline{A}}{\partial t}+\underline{\nabla} \phi \\
\text { div. } \underline{A}=-\frac{1}{c} \frac{\partial \phi}{\partial t} .
\end{gathered}
$$

The Lorentz gauge condition (5.5), when transformed to the space-independent frame, gives

$$
-\frac{\gamma_{0}^{2}}{V} \frac{\partial}{\partial T}\left(A_{Z}^{\prime}+B_{0} \phi^{\prime}\right)=-\frac{r_{0}^{2}}{c} \frac{\partial}{\partial T}\left(\phi^{\prime}+\beta_{0} A_{Z}^{\prime}\right)
$$

Since the terms contailling $A_{Z}^{\prime}$ cancel from both sides, and since a constarit poten$+i$ ii is neglected here, it turns out that

$$
\phi^{\prime}=0 \text {. }
$$

Therefore, in the $s^{\prime}$-frame the Lagrangian alid the Hanititonian are found to be

$$
\begin{aligned}
d^{\prime}= & -N_{0}\left[m_{0} c^{2}\left\{1-\frac{\gamma_{0}^{2}}{c^{2}}\left(v_{+} v_{-}+\gamma_{0}^{2} \delta v_{z}^{\prime 2}\right)\left(1-\frac{\gamma_{0}^{2}}{v} \delta v_{z}^{\prime}+\frac{\gamma_{0}^{4}}{v^{2}} \delta v_{z}^{\prime 2}-\ldots\right)^{2}\right\}^{\frac{1}{2}}+\right. \\
& +e \gamma_{0}\left(1-\frac{\gamma_{0}^{2}}{v} \delta v_{z}^{\prime}+\frac{\gamma_{0}^{4}}{2} \delta v_{z}^{\prime 2}-\ldots\right)\left\{\frac{1}{2}\left(A_{+} v_{-}+A_{-} v_{+}\right)+\gamma_{0}^{2} A_{z}^{\prime} \delta v_{z}^{\prime}\right\}+ \\
& \left.+e \gamma_{0} B_{0} A_{z}^{\prime}\right]+\frac{\gamma_{c}^{2}}{8 \pi}\left(1-\beta_{0}^{2}\right)\left(E_{+} E_{-}\right)+E_{z}^{\prime 2} \\
H^{\prime}=N_{C}\left[m_{0} c^{2}\left\{1-\frac{\gamma_{0}^{2}}{c^{2}}\left(v_{+} v_{-}+\gamma_{0}^{2} \delta v_{z}^{\prime 2}\right)\left(1-\frac{\gamma_{0}^{2}}{v} \delta v_{z}^{\prime}+\frac{r_{0}^{4}}{v^{2}} \delta v_{z}^{\prime 2}-\ldots\right)^{2}\right\}^{-\frac{1}{2}}+e \gamma_{0} B_{0} A_{z}^{\prime}\right] & -\frac{\gamma_{0}^{2}}{8 \pi}\left(1-\beta_{0}^{2}\right)\left(E_{+} E_{-}\right)-E_{z}^{\prime 2}
\end{aligned}
$$

where $A_{ \pm}=A_{x}^{\prime} \pm i A_{y}^{\prime}, E_{ \pm}=E_{x}^{\prime} \pm i E_{y}^{\prime}, v_{+}=v_{x}^{\prime} \pm i v_{y}^{\prime}$.

For a circularly polarized laser beam, we put

$$
\Sigma_{x}^{\prime}=a \cos \theta, E_{y}^{\prime}=a \sin \theta, E_{z}^{\prime}=0
$$

where $a$ is the constant amplitude of the wave, and $\theta=\omega_{ \pm}^{\prime} T$, the subscripts + and - sigris indicate the left and right circular polarized components respectively. In this case, $\varepsilon_{+}=a e^{ \pm i \theta}$, and the solution of the plasma field equations (3.1abcd)(3.9ab) is exact.

For a circularly polarized wave (5.8) arid (5.9) reduce to

$$
\Sigma^{\prime}=-N_{0}\left[m_{0} c^{2}\left(1-\frac{e^{2} a^{2} \omega_{ \pm}^{2}}{\omega_{p}^{4} m_{0}^{2} c^{2}}\right)^{\frac{1}{2}}+\frac{e^{2} a^{2}}{m_{0}^{2} \omega_{p}^{2}} j+\frac{r_{0}^{2} a^{2}}{8 \pi}\left(1-\frac{c^{2}}{v^{2}}\right)\right.
$$




$$
H^{\prime}=N_{0}\left[m_{0} c^{2}\left(1-\frac{e^{2} a^{2} \omega_{ \pm}^{\prime 2}}{\omega_{p}^{4} m_{0}^{2} c^{2}}\right)^{-\frac{1}{2}}\right]-\frac{r_{0}^{2} a^{2}}{8 \pi}\left(1-\frac{c^{2}}{v^{2}}\right)
$$

The results for the Lagrangian and Hamiltonian in the laboratory inertial frame are obtained simply by changing $\omega_{ \pm}^{\prime}$ to $\omega_{ \pm} / \gamma_{0}$ in (5.11) and (5.i2), where $\gamma_{0}^{2}=\omega_{ \pm}^{2} /\left(\omega_{ \pm}^{2}-k_{ \pm}^{2} c^{2}\right)$.

Following the method of averaged Lagrangian developed by Whitham [22-25] and exterided by cysthe [26], Das and Sihi [27-29] and others, the Lagrangian derived in this section can be used for finding the nonlinear effects including the shifts of wave parameter in the space-independent frame and then in the laboratory frame with the help of the transformation relations in Section 3.

\section{NONLINEAR DISPERSICN RELATION}

For a purely transverse circularly polarized wave,

$$
E_{ \pm}=a \exp ( \pm i \theta) \text { and } E_{z}^{\prime}=0 \text {. }
$$

So, we have

$$
P_{z}^{\prime}=0, v_{z}^{\prime}=-v_{0}+\delta v_{z}^{\prime}=-v_{0}
$$

From $(4.2 j,(4.3),(2.7 a b c),(3.13 a b c)$, and $(3.17 a b c)$, we obtain

$$
\begin{aligned}
& \dot{P}_{ \pm}^{\prime}=-e E_{ \pm}^{\prime} \pm \frac{i e V v_{ \pm}^{\prime} H_{0}}{r_{0}^{3} c\left(V-v_{0}\right)} \\
& \dot{E}_{ \pm}^{\prime}=\frac{r_{0} m_{0} \omega_{P}^{2}}{e} v_{ \pm}^{\prime} \\
& P_{ \pm}^{\prime}=m_{0} v_{ \pm}^{\prime} /\left[1-\frac{v_{0}^{2}}{c^{2}}+\frac{\left(v_{ \pm}^{\prime} \cdot v_{-}^{\prime}\right)}{c^{2}}\right]^{\frac{1}{2}}
\end{aligned}
$$

where $P_{ \pm}^{\prime}=P_{x}^{\prime}=i P_{y}^{\prime}, E_{ \pm}^{\prime}=E_{x}^{\prime} \pm i E_{y}^{\prime}, v_{ \pm}^{\prime}=v_{x}^{\prime} \pm i v_{y}^{\prime}$, and a dot denotes the derivative with respect to time.

L'sing (6.1) in (6.3) to (6.5), the nonlinear dispersion relation for the left and right circular polarization components in the space-independent frame can be obtained

$$
\frac{\omega_{ \pm}^{\prime 2}}{\left[1-\frac{e^{2} a^{2} \omega_{ \pm}^{12}}{\omega_{p}^{4} m_{0}^{2} c^{2}}\right]^{\frac{1}{2}}}-\omega_{p}^{2} \pm \frac{v \Omega_{0} \omega_{ \pm}^{\prime}}{\gamma_{0}^{3}\left(v-v_{0}\right)}=0,
$$

where $\Omega_{0}=e H_{0} / m_{0} c$. Replacing $\omega_{ \pm}^{\prime}$ by $\omega_{ \pm} / \gamma_{0}$, the dispersion relation $(6.6)$ in the laboratury frame becomes

$$
\frac{\omega_{ \pm}^{2}-k_{ \pm}^{2} c^{2}}{\left[1-\frac{e^{2} a^{2}\left(\omega_{ \pm}^{2}-k_{ \pm}^{2} c^{2}\right)}{\omega_{p}^{4} m_{0}^{2} c^{2}}\right]^{\frac{1}{2}}}-\omega_{p}^{2} \pm \Omega_{0}\left(\omega_{ \pm}^{2}-k_{ \pm}^{2} c^{2}\right)^{\frac{1}{2}}=0 .
$$

In the absence of magnetic field, $(6.7)$ becomes

$$
\omega^{2}-k^{2} c^{2}=\omega_{p}^{2}\left(1+\alpha_{0}^{2}\right)^{-\frac{1}{2}}
$$

where $\alpha_{0}$ is the dimensionless amplitude parameter ea/mowc. It is to be mentioned 
that (6.8) is identical to the results of Paul and Chakraborty [16-17]. Expanding the right-hand sice of (6.8) in power of $\alpha_{0}^{2}$ and retaining only the first two terms of biriomial expansion we obtain the results of Arons and $\operatorname{Max}$ [30] if their symbols are àdopted.

\section{FREQUENCY AND WAVE NUMBER SHIFT OF AN ELECTROMAGNETIC WAVE}

Nonline:arity in plasmas produces many interesting results in the medium and on the waves. Electromagnetic wave would have shifts in the wave parameters (wave number and frequency) as a result of norilinear interactions in plasmas [31-41]. We shall derive here the expressions for the shifts of frequency and wave number of a circularly polarized wave propagating through a cold, magnetized relativistic plasma.

(a) Frequency Shifts:

For the temporal evolution problem, we can write

$$
k_{ \pm}=k \text { and } \omega_{ \pm}=\omega_{ \pm}^{0}+\delta \omega_{ \pm}
$$

where $\delta \omega_{ \pm}$are the increments of frequencies of the left and right circular polarization components of the wave. Therefore, neglecting higher powers of $\delta \omega_{ \pm}$, we find that frequency shifts of the two polarization components are

$$
\delta \omega_{ \pm}=-\frac{\omega_{1}^{2}\left[1+\frac{\alpha^{2} \omega_{1}^{2}}{c \omega_{p}^{2}} \mp \frac{\Omega_{0}}{\omega_{1}}-\frac{\omega_{p}^{2}}{\omega_{1}^{2}}\right]}{2 \omega_{ \pm}^{0}\left[1+\frac{\alpha^{2} \omega_{1}^{2}}{\omega_{p}^{2}} \mp \frac{\Omega_{0}}{\omega_{1}}\right]}
$$

where $\omega_{1}^{2}=\omega_{ \pm}^{0^{2}}-k^{2} c^{2}$.

(b) Wave number shifts:

In the spatial evolution problem, we write

$$
\omega_{ \pm}=\omega, k_{ \pm}=k_{ \pm}^{0}+\delta k_{ \pm}
$$

where $\delta k_{ \pm}$are the increments of wave numbers of the left and right circular polarization components of the wave. Therefore, neglecting higher powers of $\delta k_{ \pm}$; the expressions for the wave number shift of the two polarization components can be derived as

$$
\delta k_{ \pm}=-\frac{\omega_{2}^{2}\left[1+\frac{\alpha^{2} \omega_{2}^{2}}{\omega_{p}^{2}} \mp \frac{\Omega_{0}}{\omega_{2}}-\frac{\omega_{p}^{2}}{\omega_{2}^{2}}\right]}{2 k_{ \pm}^{0} c^{2}\left[1+\frac{\alpha^{2} \omega_{2}^{2}}{\omega_{p}^{2}} \mp \frac{\Omega_{0}}{\omega_{2}}\right]}
$$

where $\omega_{2}^{2}=\omega^{2}-k_{ \pm}^{0^{2}} c^{2}$.

\section{DISCUSSIONS AND CONCLUSIONS}

From (7.2) and (7.5) it is observed that the intensity of the wave and static magnetic field have important roles to create the shifts of the wave parameters. It 
is seen that ( $i$ ) when $\omega>k C$ and $\omega \simeq \Omega_{0}$, the LCP components of the wave do have a frequency shift when its intensity $\alpha \neq \sqrt{2}\left(\omega_{p}^{2} / \omega^{2}\right)$, but the frequency shifts of the P.CP components exist only when $\alpha$ is greater or less than $\sqrt{2}\left(\omega_{p} / \omega\right) x$ $\left(\omega_{p}^{2} / \omega^{2}-2\right)^{\frac{1}{2}}$, and $(i i)$ when $\omega>k C$ and $\omega \simeq \omega_{p}$, the frequency shift of LCP comporicnts becclie nonzero wher $\alpha \neq\left(s_{0} / \omega\right)^{\frac{1}{2}}$, but the RCP components always have frequency shifts for all possible values of $\alpha$. The nature of the frequency shifts $0=$ RCP and LCF components of the electromagnetic wave can be well understood from Table 1 and Figures 1-3.

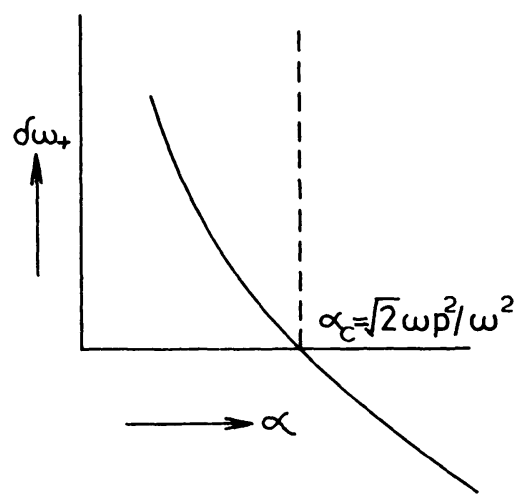

Fig. 1 Frequency shift of the LCP component V.S. Intensity of the wave (when $\omega>k C$ and $\omega \simeq s_{0}$ ).

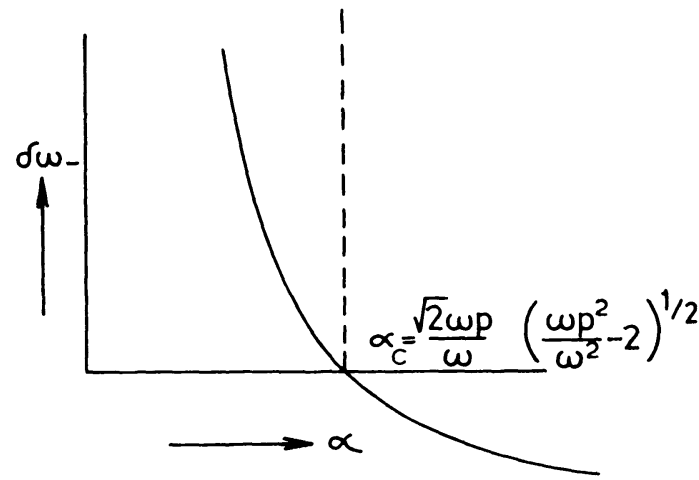

Fig. 2 Frequency shift of the RCP component V.S. Intensity of the wave (wher $\omega>k C$ and $\omega \simeq \Omega_{0}$ ).

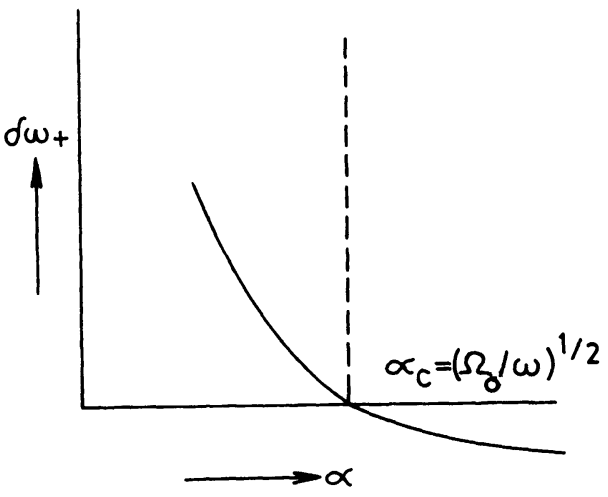

Fig. 3 Frequency shift of the LCP component V.S. Intensity of the wave (when $\omega>k c$ and $\omega \simeq \omega_{p}$ ).

\section{NUMERICAL ESTIMATION}

For the rudiations of Nd-glass laser having wave length $=1.06 \mu \mathrm{m}$, frequency $=$ $1.78 \times 10^{15} \mathrm{c} / \mathrm{s}$, Fower $=10^{16}$ watts $/ \mathrm{cm}^{2}$ (which is less than the threshold power to generate self-action effects) passing through a dense plasma $\left(N_{0}=10^{20} /\right.$ c.c.), the irequency shift of LCP components $=1.01 \times 10^{15} \mathrm{c} / \mathrm{s}$ and that of $R C P$ components = $-8.6 \times 10^{14} \mathrm{c} / \mathrm{s}$ when $\omega \simeq \Omega_{0}$, i.e., Ho $\simeq 10^{8}$ gauss.

Under the situation $\omega \simeq \omega_{p}$, the frequency shift of the LCP and RCP components become $4.42 \times 10^{12} \mathrm{c} / \mathrm{s}$ arid $4.31 \times 10^{12} \mathrm{c} / \mathrm{s}$ respectively when $H_{0}=10^{5}$ gauss. For magnetic field $\simeq 10^{6}$ gauss, the frequency shift of the LCP and RCP components become $3.48 \times 10^{12} \mathrm{c} / \mathrm{s}$ and $5.22 \times 10^{12} \mathrm{c}$ 's respectively. 


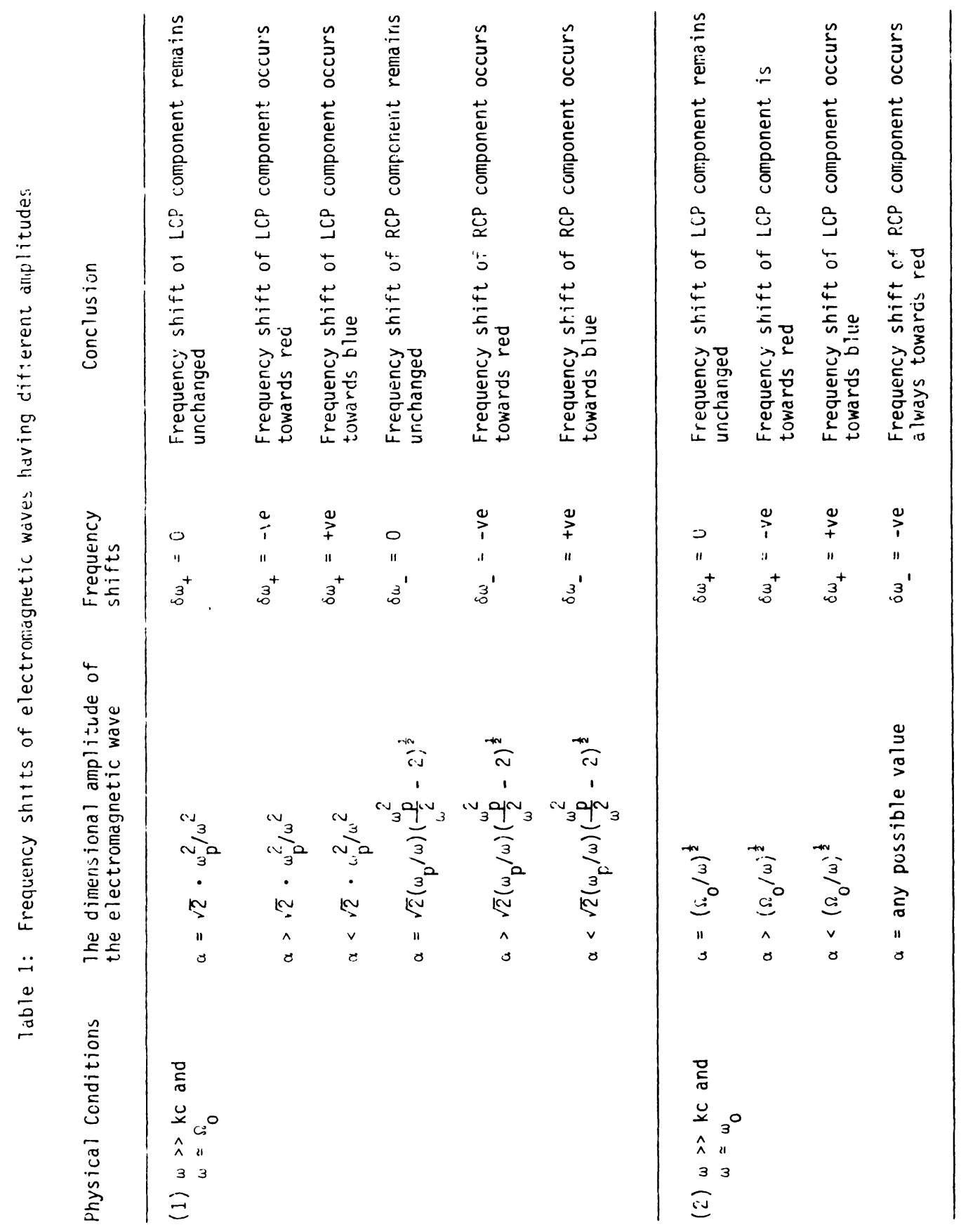

10. CLOSING REMARKS

Transformation of field variabies from the laboratory frame to the spaceindependent frame is applicable only for a single electromagnetic wave because the coridition required for this transformation, i.e., $v_{0}=k c^{2} / w=c^{2} / V$ is not satisfied for two or more waves interacting in plasma.

To derive the results for the shifts of wave parameters with the help of special Lorentz transfornation, fiela variables should be transformed to the space- 
inciependent frame at the beginning, and then one should proceed with the calculation. If equations are solved in the laboratory frame before transforming then to the space indeperident frame and transformations are used only in the subsequent steps, correct results would not appear in the space-independent frame.

\section{ACKNCWLEDGEMENTT}

This work was partidi?y supported by the University of Central Florida.

\section{REFERENCES}

[ 1] Winkles, B.B. and Eldridge, 0 ., Self-consistent electromagnetic wave in relativistic Vlasov plasma, Phys Fluids 15 (1972) 1790-1800.

[2] Clemmow, P.C., Nonlinear waves in a cold plasma of Lorentz transformation, J. Plásma Phys. 12 (1974) 297-317.

[3] Clemmow, P.C., Nonlinear superluminous, periodic waves in a plasma with magnetic field, J. Plasma Phys. 17 (1977) 301-316.

[ 4] Chian, A.C.L. and Clemmow, P.C., Nonlinear periodic waves in a cold plasma: a quantitative analysis, J. Plasma Phys 14, (1975) 505-527.

[5] Kennal, C.F. and Pellat, R., Relativistic ronlinear plasma waves, in a magnetic field, J. Plasma Phys. 15, (1976) 336-355.

[6] Shih, L.Y., Nonlinear interaction of a magnetized overdense plasma with relativistically strong electromagnetic waves, J. Plasma Phys. 20, (1978) 289-298.

[ $7 j$ Decoster, A., Nonlinear travelling waves in a homogeneous cold collisionless plasma, Phys. Reports 47, (1978) 285-422.

[ 8] Lee, M.A. and Lerche, I., On the stability of self consistent large amplitude waves in a cold plasma, Part-1, Transverse circuarly polarized waves in the absence of large scale magnetic field, J. Plasma Phys. 20, (1978) 313-328.

L9] Lee, M.A. and Lerche, I., On the stability of self consistent large amplitude waves in a cold plasma, Part-2, Longitudinal waves in the absence of large scale magnetic field, J. Plasma Phys. 21, (1979) 27-42.

[10] Lee, M.A. and Lerche, I., On self-consistent waves and their statility in warm plasma, Part-1, Construction of self consistent waves, J. Plasma Phys. 21, (1979) $141-150$.

[11j Lee, M.A. and Lerche, I., On self consistent waves and their stability in warm ilasma, Part-2, Instability of circularky polarized waves hoth in the presence and absence of an ambient magnetic field, J. Plasma Phys. 24, (1980) 89-102.

[12] Clemmow, P.C. and Harding, R.D., Nonlinear waves in a magnetized plasma, periodicity analysis, ‥ Plasma Phys. 23 (1980) 71-90.

[13] Akhiezer, A.I. and Polovin, R.V., Theory of wave motion of an electron plasma, Sov. Phys. JETP, 3, (1956) 696-705.

[14] Wong, H.S.C., Nonl inear stationary waves in relativistic plasmas, Phys. Fluids 6, (1963) 1115-1123.

[15] Wong, H.S.C., Nonlinear transverse wave in plasmas, Phys. Fluids $\underline{6}$, (1963) $1458-1464$.

[16] Paul, S.N. and Chakraborty, B., Some applications of transformation to the space-independent frame in the process of strong radiation in plasmas, Austr. J. Phys. 36, (1983) 67-77.

[17] Paul, S.N. and Chakraborty, B., Some aspects of transformation of the nonlinear piasma equations to the space-independent frame, Physica C122, (1983) 139-149. 
[13] Akhiezer, A.I., Akhiezer, I.A., Polovin, R.V., Sitenko, A.G., Stepanov, K.N., Plasma Electrodynamics, Pergamon Press, New York, Vol. 2, Chap. 8 (1975).

[19] Boyd, T.J.M. and Sanderson, J.J., Plasma Dynamics, Neison, London, p. 27 (1969).

[20] Hughes, W.F. and Young, F.J., The Electrodynamics of Fluids, Wiley, New York, p. 19 (1966).

[21] Landau, L.D. and Lifshitz, E.M., The Classical Theoty of Fields, Pergamon Press, Article - 75, p 209 (1975).

[22] Whitham, G.R., Nonlinear dispersive waves, Proc. Roy. Soc. London, A283, (1965) $238-261$.

[23j Whitham,. G.E., A gererd] approach to linear and nonlinear dispersive waves usirs a Largangian, J. Fluid Mech. 22, (1965) 273-283.

[24] Whitham, G.B., Variational methods and applications to water waves, Proc. Roy. Soc. Loridon, A299, (1967) 6-25.

[25] Whithäll, G.B., Linear and Nonlınear Waves, John Wiley, New York (1974).

[26? Dysthe, K.B., A note on the application of Whithan's method to nonlinear waves in dispersive media, J. Plasma Phys. 11 (1974) 63-76.

[ai] Das, K.P. and Sihi, S., Modulationâl instability of elliptically polarized transverse electromagnetic wave in a cold plasma, Czech. J. Phys. B27, (1977) 1368-1377.

[28] Das, K.P. and Sihi, S., Modulational Instability of two transverse waves in a cold plasma, Plasma Phys. 21, (1979) 183-192.

[29] Das, K.P., and Sihi, S.,Norilinear resonant interaction of three extraordinary waves in a hot electron plasma, Beitrage aus der plasma physik, 20, (1980) $215-223$.

(30] Arons, J. and Max, C.E., Self precession and frequency shift for electromagnetic waves in humogeneous plasmas, Phys. Fluids, 17, (1979) 1983-1994.

[31] Sturrock, P.A., Nonlinear effects in electron plasma, Proc. Roy Soc. London, A242, (1957) $277-i 99$.

[32] Dawscr, J., Nonlinear oscillations in a cold plasma, Phys. Rev. 113, (1959) 383-387.

[33] Jacksorı, R.A., Nonlinear oscillations in a cold plasma, Phys. Fluids $\underline{3},(1960)$ $831-832$.

[34] Montgomery, D. and Tidman, D.A., Secular and nonsecular behaviour for the cold plasma equations, Phys. Fluids I, (-1964) 242-249.

[35] Sluijter, A.W. and Montgomery, D., Comments on secular and non-secular behaviour for the cold plasma equations, Phys. Fluids $\underline{8}$, (1965) 551-552.

[36] Tidman, D.A. and Stainer, H.W., Frequency and wave number shifts for nonlinear waves in hot plasma, Phys. Fluids 8 (1965) 345-353.

[37] Das, K.P., Nonlinear effects of propagating extraordinary waves in a cold plasma, Phys. Fluids 11 , (1968) 2055-2057.

[38] Das, K.P., Nonlinear effects of two transverse monochromatic waves in a cold plasma, Phys. Fluids 14, (1971) 2235-2237.

[39] Boyd, T.J.M., Nonlinear extraordinary waves in a cold plasma, Phys. Fluids 10, (1967) 896-898.

[40] Goldstein, S. and Salu, Y., On a family of exact nonlinearly waves in plasmas, J. Plasma Phys. 10, (1973) 231-235. 
[41] Schindler, K. and Janicke, L., Large amplitude electromagnetic waves in hot relativistic plasmas, Phys. Letts. A45, (1973) 91-92.

*Permanent Adiciress of S. N. Paul:.

13, T. C. Goswami Street

Serampore, Hooghly

Pin 712201

West Eerigal, India. 


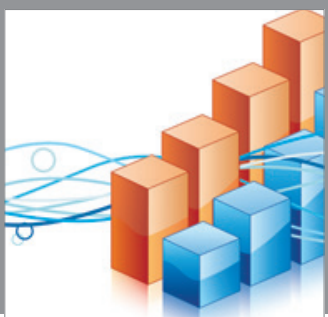

Advances in

Operations Research

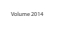

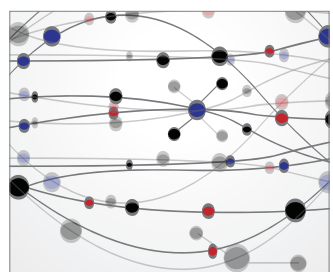

\section{The Scientific} World Journal
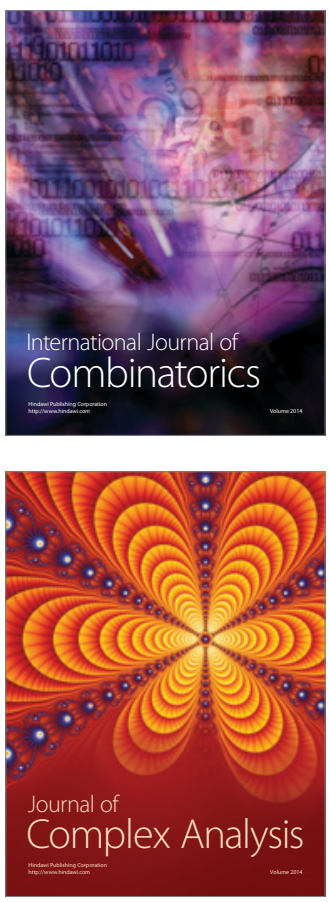

International Journal of

Mathematics and

Mathematical

Sciences
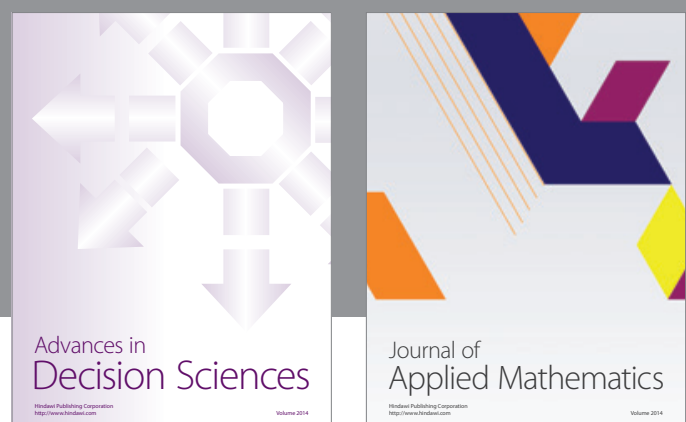

Journal of

Applied Mathematics
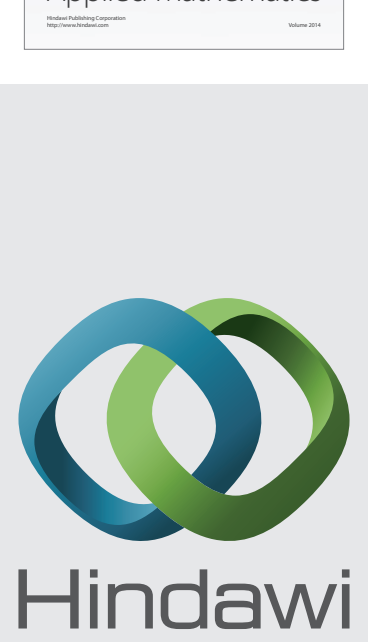

Submit your manuscripts at http://www.hindawi.com
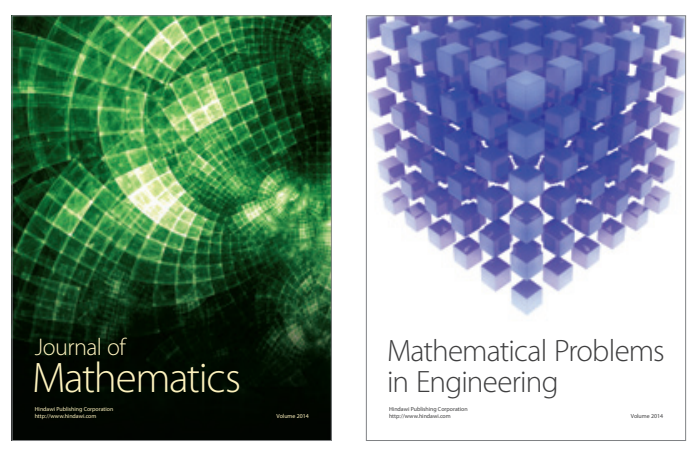

Mathematical Problems in Engineering
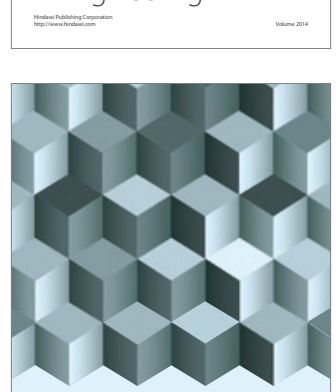

Journal of

Function Spaces
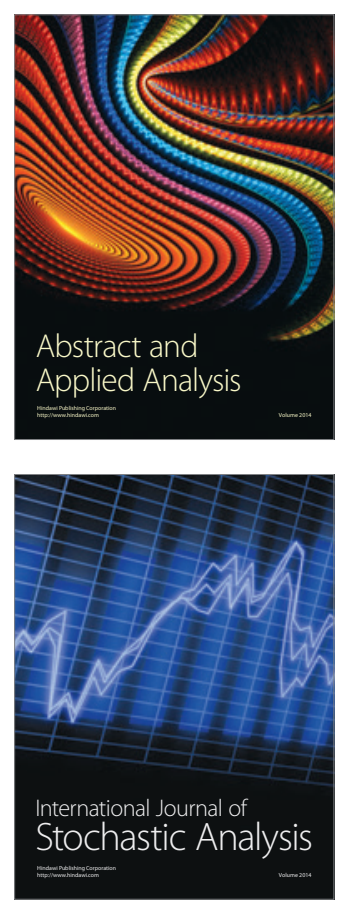

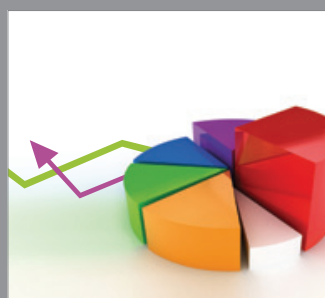

ournal of

Probability and Statistics

Promensencen
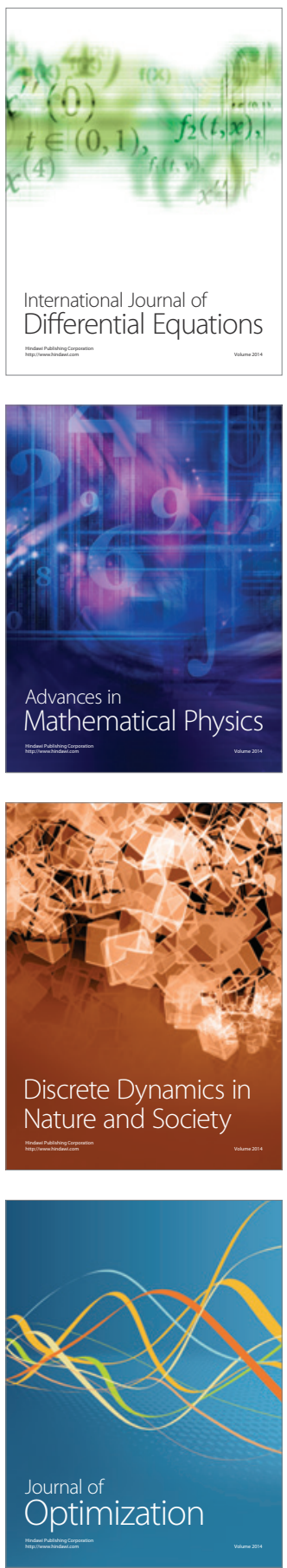